\title{
Ingresos laborales, transferencias y seguridad económica de los adultos mayores de Medellín, Colombia
}

\author{
Labor income, transfers and economic security for the elderly of Medellin, Colombia
}

\author{
Sonia Isabel Aguilar-Fuentes', Doris Cardona-Arango²
}

\begin{abstract}
1 Mg. en Administración. Licenciada en Ciencias de la Comunicación, Universidad Autónoma de Chihuahua, México. e-mail: sonyacomunicacion@gmail.com

2 PhD. en Demografía. Docente Universidad CES. Medellín, Colombia. e-mail: doris.cardona@gmail.com
\end{abstract}

Aguilar-Fuentes SI, Cardona-Arango D. Ingresos laborales, transferencias y seguridad económica de los adultos mayores de Medellín, Colombia. Univ. Salud. 2016;18(1):34-45. DOI: http://dx.doi.org/10.22267/rus.161801.17

\section{Resumen}

Objetivo: Explorar la seguridad económica de los adultos mayores en Medellín, Colombia, así como sus fuentes de ingreso y transferencias económicas, desde una mirada comprensiva. Materiales y métodos: Estudio cualitativo exploratorio de fuente primaria, con selección por bola de nieve por medio del método histórico-hermenéutico. Se entrevistaron quince adultos mayores y cinco especialistas en el tema, a los cuales se les indagó por aspectos relacionados con sus condiciones económicas y se analizó la información, como una teoría fundamentada, donde emergen categorías de los textos. Resultados: Los mayores se sienten inseguros económicamente al no contar con ingresos fijos y suficientes que les permitan vivir de manera autónoma e independiente; menos de una tercera parte cuenta con ingresos provenientes de un empleo, el cual es informal en la mayoría de los casos Por otro lado, las transferencias por pensión y jubilaciones están determinadas por la ocupación y tipo de vinculación en su anterior vida laboral y muchos de ellos deben recurrir a las transferencias económicas de sus familiares. Conclusión: Los adultos mayores entrevistados ven comprometida su seguridad económica por no contar con un ingreso económico para la cobertura de necesidades básicas, ya que una gran mayor parte de ellos carecen de ingresos fijos, que los lleva a la informalidad para vivir el día a día, conscientes de que cada vez se envejecerá más, aumentará la dificultad para seguir laborando y se reducen los espacio para laborar y por ello deben depender del Estado o la familia.

Palabras clave: Seguridad social; adulto mayor; salarios y beneficios. (Fuente: DeCS, Bireme).

\begin{abstract}
Objective: To explore the economic security of the elderly in Medellin, Colombia as well as their sources of income and economic transfers from a comprehensive perspective. Materials and methods: An exploratory qualitative study of primary source with selection by snowball through historical hermeneutic method was conducted. 15 seniors and 5 specialists in the field selected by snowball were interviewed and asked for aspects related to their economic conditions. The given information was analyzed, as a grounded theory, where categories from the texts emerge. Results: Elderly people feel insecure financially because they do not have fixed and sufficient income to live autonomously and independently. Less than one-third have income from employment, which in most cases is informally. On the other hand, transfers from pension and retirement benefits are determined by the occupation and type of labor relationship in their previous jobs and many of them must rely on financial transfers from their families. Conclusion: Elderly people, who were interviewed, see their economic security compromised by not having an income to cover basic needs, since a large majority of them lack of fixed incomes. This situation leads them to work informally to live day to day knowing that every time they are getting older, it is more difficult to continue working, the job opportunities are reduced and therefore they must rely on the State or their families.
\end{abstract}

Keywords: Social security; elderly people; wages and benefits. (Source: DeCS, Bireme). 


\section{Introducción}

El presente artículo es un análisis de la seguridad económica de los adultos mayores de Medellín, Colombia, y de las fuentes de ingresos laborales, así como de las transferencias familiares, jubilación y pensión, y los programas sociales, es decir, las fuentes de ingreso no laborales. La seguridad económica en la vejez, definida como la capacidad de disponer y usar de manera independiente recursos económicos, es un factor importante para lograr la calidad de vida. Para su estudio, es necesario hacerlo desde la posición y la situación económica.1,2

La situación económica de las personas adultas mayores está determinada por su poder adquisitivo, que se sustenta con ingresos de diversas fuentes, sean estas laborales o no laborales.2 Las fuentes laborales son las que provienen como retribución a un trabajo, en tanto que las no laborales tienen que ver con transferencias económicas, las cuales pueden ser familiares, pensión y jubilación o programas sociales entre otros.3 Es la seguridad económica una legítima aspiración y en ella influyen factores como ciclo vital individual y familiar, las biografías personales, características de los sistemas de protección social, así como las biografías generacionales. 2

En contraparte de la seguridad económica existe la inseguridad económica que según la Organización Internacional del Trabajo (OIT), es multidimensional al igual que la pobreza.4 Para efectos de este trabajo, se medirá la situación económica de los adultos mayores desde la seguridad y la inseguridad. Por otro lado, se aborda el emprendimiento como aquella actividad que inicia el adulto mayor con fin de lograr ingresos económicos para subsistir, arrojando como resultado su autoempleo, y al igual que las ocupaciones laborales, puede darse desde la formalidad o la informalidad.5 Para efectos de este trabajo, se toma el emprendimiento como autoempleo pues es la realización de una actividad con la intención de lograr ingresos económicos; la mayoría de las veces informal, puesto que vivimos en una sociedad que tienes como matriz cultural al productivismo y que asume a los adultos mayores como un estorbo y carga financiera. 6,7

Es importante tomar en cuenta la percepción de los adultos mayores respecto al tema que se investiga, es decir lo que ellos consideran respecto a su situación económica actual, pues esto permite tener una visión integral de sus condiciones de vida, circunstancias materiales, psicológicas y emocionales, así como la valoración que tienen sobre las mismas.8

Debido a que el proceso de envejecimiento es normal, natural e inevitable, es urgente reflexionar acerca de la condición actual de quienes lo viven, por ello se buscó profundizar en la seguridad económica de los adultos mayores en Medellín, Colombia, así como sus fuentes de ingreso y transferencias económicas, pero desde una mirada más comprensiva, indagando a los directamente implicados.

\section{Materiales y métodos}

Bajo un enfoque cualitativo se realizó un estudio exploratorio de fuente primaria, seleccionados por bola de nieve usando el método históricohermenéutico, mediante el que se analizaron las respuestas de entrevistas a personas mayores de 60 años de la ciudad de Medellín, Colombia, y de especialistas en el tema del adulto mayor. A través de entrevistas semiestructuradas realizadas entre junio y julio de 2013, a los cuales se les indagó sobre su percepción sobre la seguridad económica con que cuentan los adultos mayores de la ciudad, para responder a sus necesidades, así como el fenómeno de las transferencias económicas que se dan entre las familias.

Se entrevistó a 15 adultos mayores en un rango de edad de los 61 a los 90 años, a quienes se tuvo acercamiento a través de una institución educativa de nivel universitario con programa educativo para mayores de 55 años, dos Centros de Protección Social, así como espacios públicos y las calles de esta localidad. Los criterios de inclusión para las personas mayores fueron el tener más de 59 años de edad, y sólo se excluyó a personas con deterioro cognitivo para responder a las preguntas, evidente para la entrevistadora, 0 
bien, que no quisieran participar de las entrevistas. También se entrevistó a cinco funcionarios de gobierno, y especialistas involucrados de manera directa o indirecta en la realización de actividades encaminadas al mejoramiento de las condiciones de vida y seguridad social del adulto mayor. El criterio para entrevistar a especialistas fue el que tuvieran experiencia en el trabajo directo con adultos mayores o conocimiento especializado en la materia. Asimismo se pidió que tuvieran disposición en participar de la entrevista.

Con las categorías de interés inicial y que responden al objetivo planteado: situación económica actual, fuentes de ingreso laboral y no laboral, apoyos y subsidio del estado, percepción sobre la situación económica y transferencias generacionales, se construyó una guía de entrevista las cuales fueron aplicadas por una de las investigadoras, previa autorización de grabación y seguidamente se transcribieron en su totalidad; se contó con el consentimiento verbal de cada uno de los participantes y se consideraron los principios éticos de la investigación, contemplados en la Resolución 008430 de 1993. Con ellas se analizó la información, como una teoría fundamentada, donde emergen categorías de los textos, desde las categorías iniciales abiertas hasta las selectivas, donde se conformaron conceptos más diferenciados que son los que se exponen en los resultados.

\section{Resultados y discusión}

\section{Concepto de seguridad económica en la vejez, según los entrevistados}

Los adultos mayores entrevistados para la realización del presente análisis viven en distintas situaciones y condiciones, de acuerdo a las cuales cada uno manifestó sentirse seguro, inseguro, más o menos o tranquilo. Quienes se declaran seguros económicamente no necesariamente perciben ingresos altos, sin embargo tienen en común el ser independientes, que toman decisiones sobre su vida -e incluso sostienen a sus familiares- $y$ controlan sus gastos, esto en congruencia con lo que señala la teoría: todo esto corresponde a resultados.
"Yo me siento muy seguro, trabajo de lunes a viernes y me alcanza con lo que gano para mí y mi señora, estoy muy tranquilo" (AM11, comunicación personal, 1 julio de 2013) esto es un hallazgo.

" (Me siento) muy segura, muy segura salvo que ocurra alguna cosa pero por ahora muy segura, yo manejo muy bien los centavitos que tengo, no malgasto, no hago inversiones extravagantes, tengo mi casa muy buena, tengo mi carrito, tengo lo que necesito... tengo muchas cositas que son alquiladas, propiedades que están en renta, esto me lo manejan en la agencia de arrendamiento y yo mensualmente voy por mi dinero y lo tengo muy fijo, esto es para esto, para esto y me sobra obviamente" (AM1, comunicación personal, 18 de junio de 2013).

Por otro lado, los mayores que no cuentan con un ingreso fijo o suficiente señalan sentirse inseguros. Se trata de personas que trabajan, pero sus ingresos no alcanzan a cubrir sus gastos, y también de beneficiarios de algún CPSAM, donde si bien tienen alimento y alojo seguro, ya no gozan de la independencia económica que tuvieron mientras trabajaron. Ambos grupos de adultos, están limitados en su toma de decisiones y en su nivel adquisitivo:

"Me preocupa (situación económica), sinceramente sí pues a uno le hace falta cualquier peso en el bolsillo porque a veces a uno se le antoja una cosa y no tiene uno con qué comprarla. Entonces le hace falta a uno" (AM4, comunicación personal, 21 de junio de 2013).

"Si sale a la calle no tiene para tomarse un tinto en el parque Berrio ni en ninguna parte y eso sí como que lo va acomplejando a uno, y se va uno como estresando algo, dando gracias a Dios que tiene uno lo más importante, que es la comida, la bebida, el arreglito de la ropa, esas cosas" (AM6, comunicación personal, 21 de junio de 2013).

Los adultos mayores que manifestaron sentirse más o menos con respecto a su seguridad económica, de manera sorpresiva son aquellos que cuentan con ingresos fijos y suficientes para sostener su vida actual según lo manifiestan ellos 
mismos, sin embargo, les genera incertidumbre lo que pudiera suceder a nivel macroeconómico.

"He vivido tranquilamente, me he sentido bien, no necesito trabajar para seguir viviendo... Entonces, hoy estoy tranquilo, hay que seguir pensando qué va a pasar mañana con mi jubilada, cuáles van a ser los cambios laborales de Colombia que te van a seguir afectando" (AM3, comunicación personal, 18 de junio de 2013).

Contrario a lo anterior, pasa en otro grupo de adultos mayores que a pesar de no contar con un ingreso ni fijo ni suficiente, manifiestan sentirse tranquilos. Estos adultos tienen en común que ya no se sienten con fuerza para desempeñar algún trabajo, o bien, durante su juventud no laboraron, sino que fueron de apoyo para la familia y hoy son beneficiarios del estado y permanecen en algún CPSAM, donde afirman, no tienen que ocuparse por buscar su sustento.

"Plata no hay, pero aquí lo tiene uno todo. Gracias a Dios". (AM8, comunicación personal, 26 de junio de 2013).

Expertos en el tema del adulto mayor en Medellín, coinciden que no sólo a nivel municipal sino nacional, prevalece la inseguridad económica en el grupo etario en observación puesto que gran porcentaje de ellos no tiene acceso a un ingreso fijo y constante que le permita vivir con calidad. Lo anterior, se deriva principalmente de que en su juventud trabajaron en la informalidad y de que hoy no existan los mecanismos para que los mayores reciban un subsidio digno por el sólo hecho de ser mayores.

"La situación económica de los adultos mayores hoy está comprometida, muy comprometida porque no tienen ni para sostenerse ellos mismos" (EI5, comunicación personal, 12 de julio de 2013).

"A nivel de Colombia todavía no hemos llegado a un punto donde las personas mayores pueden demostrar niveles de ingresos que les permitan solventar su existencia... si hablamos de personas en un rango de los 70 años por lo general no tienen jubilación porque en el tiempo en que trabajaron no creyeron en el sistema del Seguro Social, se dedicaron a actividades que provenían de pronto de la conformación de comercios, y había la creencia de para qué aportar" (EI1, comunicación personal, 14 de junio de 2013).

"La realidad económica no solo en Medellín, en Colombia, para las personas mayores es realmente lamentable. Muchos de nuestros viejos no pueden acceder a una pensión, muchísimos de ellos se quedaron en trabajos que no estaban siendo calificados, no se asumió su labor... nuestras viejas, nuestras mujeres, muchas fueron trabajadoras domésticas por años en una equis pero nunca le fue valorado su hacer desde la legalidad, desde la norma, y no accedieron a una jubilación" (EI3, comunicación personal, 26 de junio de 2013).

La seguridad económica en la vejez, es considerada como los recursos económicos de que dispone la persona mayor, agrega calidad a los años pues permite la autonomía, mejoramiento en la autoestima, propicia el desempeño de roles significativos y la participación en la vida cotidiana como ciudadanos con plenos derechos.2 Comprende pues la posición económica y la situación económica. La posición económica se evalúa según los ingresos obtenidos por los mayores en relación con otros grupos o con la población total; la situación económica tiene que ver más con el poder adquisitivo mediante el recurso que puede provenir de distintas fuentes. 2

En contraparte existe la inseguridad económica, que según la Organización Internacional del Trabajo (OIT), es multidimensional al igual que la pobreza, pues se relaciona con el riesgo, la capacidad de hacer frente a las consecuencias del mismo y a la capacidad de recuperación.4

\section{Fuente de ingresos de los adultos mayores}

Mediante las entrevistas realizadas se encontró que una parte importante ( 8 de 15 ) de adultos mayores tienen en el campo laboral su principal fuente de ingreso. Este grupo de mayores tiene en común que son personas que no cuentan con jubilación o pensión, ni otra forma de ingreso que les contribuya a su sostenimiento. 
Los mayores, consideran que a su edad es difícil o imposible encontrar un empleo formal, señalando que su edad, falta de preparación escolar y la competencia con los jóvenes son las principales causas de la negativa. Por lo anterior, la mayoría de quienes trabajan lo hacen desde la informalidad, situación presente en sus biografías personales desde la juventud. Las principales actividades son la venta de fruta, ropa $y$ accesorios, así como elaboración y venta de manualidades. Estos mayores encuentran en su trabajo el único medio de ingreso, que desde la informalidad es bajo, sin embargo, esa es la única opción que tienen para sostenerse.

"Hay veces que no vendes mayor cosa, o no se vende nada. Pongámosle, más o menos 25 mil pesos... y aquí hay que fregarle aunque sea ganando poquito, o ganando lo que sea porque yo no me puedo quedar sentado en una casa" (AM14, comunicación personal, 9 de julio de 2013).

"Hago tapetes, los hago por encargo. Por ejemplo, usted me encarga uno y si no tengo material, le digo deme tanto y el día que se lo entregue, me da tanto. Si tiene afán me dedico más y lo termino" (AM8, comunicación personal, 26 de junio de 2013).

Otro aspecto obtenido en las respuestas, es que algunos adultos mayores continúan trabajando para mantenerse activos, y aunque hacen uso de los ingresos que logran, la obtención de éstos no son trascendentales en su estilo de vida. Cabe señalar que estos casos son menos.

"Yo sigo ejerciendo derecho porque yo soy abogado de la senectud, yo terminé derecho por ahí a los 50 años... pero pues sigo todavía ejerciendo porque hay que buscarle todavía, las necesidades económicas algunos no las satisfacemos del todo" (AM2, comunicación personal, 18 de junio de 2013). "Es muy relativa la venta... hay semanas en que uno hace 200, hace 100, otra semana hace por ahí 50, y así... yo fui maestra, yo soy jubilada y soy licenciada. Trabajo ahí por no quedarme en la casa. Ay, no. Yo no me amaño en la casa" (AM13, comunicación personal 15 de junio de 2013).

Especialistas señalan que ante el panorama colombiano, donde a los 35 años ya se está en edad límite para encontrar trabajo, no existen las condiciones para que los adultos mayores trabajen con el goce de las garantías laborales; los adultos mayores que deseen trabajar están destinados a hacerlo desde la informalidad, al no existir apertura en las empresas ni impulso desde los gobiernos para que el mayor pueda ejercer su derecho al trabajo.

"En la parte de informales hay un gran sector que son mayores, no tienen de dónde más, no estuvieron educados ni capacitados para acceder a un sistema que todos los días está exigiendo más excelencia y ellos por su condición de una generación donde el máximo nivel educativo era hasta secundaria" (EI5, comunicación personal, 12 de julio de 2013).

Lo anterior, pese a que desde las instancias de gobierno se habla de la necesidad y búsqueda de entornos favorables que lleven al mejoramiento de las condiciones de vida, mayor grado de bienestar de la población y a garantizar el envejecimiento activo con condiciones de autonomía, integración, seguridad y participación efectiva de la población en todo su ciclo de vida.14

"Una persona adulta en Colombia no tiene que ser mayor de 60 años, aun desde los 35, 40 años ya no puede ingresar a una empresa... el adulto mayor nuestro, permeado por esa visión de las sociedades occidentales, empieza a verse relegado, como una carga, como el que demanda el servicio, consume recursos, el que se le da mucho y no aporta nada" (EI5, comunicación personal, 12 de julio de 2013).

"En estos momentos en Colombia yo no veo actividades laborales en los adultos mayores independientemente de que una persona mayor pueda ser propietaria de su propio negocio, yo no la veo porque estamos en un país con una composición de jóvenes bastante elevada que van reclamando su puesto y también tenemos como una cantidad de creencias alrededor de un viejo trabajando. Las actividades independientes son las que más caracterizan el trabajo de las personas mayores que por obligación tienen que trabajar" (EI1, comunicación personal, 14 julio de 2013).

La situación económica en el adulto mayor, como parte de la seguridad económica tiene que ver con 
su poder adquisitivo.2 Sus fuentes de ingreso pueden ser laborales o no laborales. Los ingresos laborales son los que provienen como retribución a un trabajo realizado, en tanto que los ingresos no laborales pueden ser transferencias económicas, rentas de propiedades y otros ingresos extraordinarios que pudiera recibir.9 Así, a medida que avanzan los años, las fuentes de ingreso de los individuos varían; en la juventud proviene de un salario; en el ciclo intermedio de utilidades por la explotación de negocios propios (incluido el trabajo por cuenta propia), y en la etapa avanzada predominan las transferencias, es decir, aportes en dinero en efectivo.10

Como parte de la seguridad económica los ingresos son fundamentales para construir y mantener calidad de vida, así como seguridad presente y en perspectiva. Son un medio para acceder a diferentes oportunidades, mantener cierta autonomía y participar en la sociedad; por el contrario, la falta de ingresos se asocia con condiciones de vida precaria familiar e individual.11 $_{\text {. }}$

En Colombia alrededor del $27 \%$ de las personas mayores de 59 años trabaja y el 70 por ciento lo hace desde la informalidad.12

En el caso del adulto mayor hombre, más del 70\% trabaja lo hace por cuenta propia, el 13\% son peones, jornaleros o trabajadores familiares sin pago, y el $11 \%$ son obreros o empleados de empresas. Por su parte, de las mujeres trabajadoras el $71 \%$ lo hace por cuenta propia, $9 \%$ son empleadas domésticas y el $8 \%$ obreras o empleadas de empresa.12 Hoy los adultos mayores se enfrentan a una generación de jóvenes que inicia su vida laboral, por lo que es común encontrar a las personas mayores trabajando en la informalidad.13

El lograr que un adulto mayor continúe trabajando significa convencer a todo un sistema económico y social de que los mayores actúan distinto a los jóvenes en determinadas situaciones laborales, sin embargo esto no significa menor capacidad.14

En la ciudad de Medellín el comercio informal es visible en toda la ciudad y entre quienes lo ejercen se encuentran a personas de todas las edades; los adultos mayores aun en este rango compiten con otros más jóvenes, quedando en evidente desventaja debido a su menor fuerza física, rapidez, entre otros factores.

La cifra local señala que el 50,09 \% de los trabajadores de todas las edades lo hace desde la informalidad, porcentaje que es uno de los menores del país, si se toma en cuenta al más alto porcentaje con el 84,1 \% en Quibdó.15

De otro lado, en Colombia los adultos mayores que no se encuentran laborando, dan como razones principales para no hacerlo su estado de salud, que ya están jubilados o que sienten que ya no consiguen trabajo.12 Dichos adultos, podrían encontrar su sustentabilidad en fuentes no laborales de ingreso, entre las cuales se encuentran las transferencias económicas, que se dividen en envíos de dinero dentro o fuera del país, jubilación por sistemas de pensiones, y los ingresos mediante programas sociales.9

\section{Transferencias familiares}

El levantamiento de información indica que sólo una pequeña parte de los adultos mayores recibe apoyo de las redes familiares. Quienes manifiestan no contar con jubilación, pensión o trabajo, afirman también que en su familia no encuentran apoyo para la cobertura de sus necesidades. Cabe señalar que en la mayoría de estos casos existe desapego por desintegración en las familias y el adulto mayor vive prácticamente olvidado.

Aquellos que sí gozan de una aportación familiar, están divididos entre quienes cuentan con este tipo de transferencia como única fuente de ingreso, $\mathrm{y}$ entre quienes lo reciben como complemento a sus pensiones. En el primer caso, se trata de adultos que residen en CPSAM y este ingreso proviene principalmente del único lazo familiar que mantiene, el cual resulta ser con hermanos o sobrinos. En el caso de los hermanos, también se trata de personas de la tercera edad que viven en situación de pobreza, por lo que el beneficio se torna irregular e insuficiente y es el adulto mayor quien en varias ocasiones tiene que 
pedirlo o bien recurrir a otras actividades para obtener dinero, aunque sea ocasionalmente.

"Un hermano mío de Sabaneta y en el trascurso de un mes vino 4 veces, me daba poquita plata, poquita pero me daba... ahorita ya no. Hará como mes y medio me dijo que le iban a hacer una operación y le dije me hace una llamadita y si yo tengo forma lo voy a visitar a la clínica, pero si yo no tengo plata pues ni que me avise si no puedo. $Y$ no, nadie más, por ahí tengo sobrinos pero por acá no se asoman, nadie aparece" (AM6, comunicación personal, 21 de junio de 2013).

"Tengo un hermanito que me colabora, pero a mí me da pena, porque es el único, las hijas no llegan a venir, Mi hermano es el que me sostiene la droga pero ya me da pena... el sábado salí a pedir y junté seis mil pesos y con eso compré dos cajas de pastillas" (AM7, comunicación personal, 26 de junio de 2013).

En el caso de quienes reciben transferencias familiares como ingreso adicional a otras fuentes, manifestaron recibirlas de sobrinos o hijos. Este tipo de apoyo es más constante y podría derivarse de la condición en que viven los benefactores, que por lo regular son profesionales.

"Yo tengo un hijo en el exterior. Tengo tres en el exterior pero uno es (como) mi maridito que me mantiene como una reina. Me compró casita y vivo en una unidad preciosa" (AM12, comunicación personal, 9 de julio de 2013).

También es de destacarse que el adulto mayor es muchas veces quien emite una transferencia principalmente en apoyo a sus hijos; estas personas tienen en común el contar con una pensión por jubilación, la cual llega a ser incluso insuficiente ante este apoyo que dan.

La fuente de ingresos a través de la familia para el adulto mayor de Medellín fue una de las menos mencionadas por especialistas consultados. Lo anterior podría reflejar lo que las estadísticas señalan en Colombia poco más del 30\%.

Para quienes si encuentran en su familia una fuente importante de apoyo a su economía, este puede ser el principal medio de subsistencia, afirman especialistas. Sin embargo, esto también significa permanecer en una situación vulnerable pues dicha entrada económica está sujeta, por otro lado, a los ingresos de quien la otorga.

"Vemos que muchos (ingresos) se conforman gracias a la ayuda de sus hijos, que vienen a aportar para que el papá tenga un nivel de ingresos... que en muchas personas mayores, en un momento de la existencia pueden tener dificultades económicas porque sucede algo con su nivel de ingresos que puede ser que el de sus hijos disminuya y esto viene a afectarlos" (E1, comunicación personal, 14 de junio de 2013).

Como un reflejo de lo que ocurre a nivel Colombia, del total de los adultos mayores entrevistados, sólo un porcentaje mínimo cuenta con jubilación, producto de una vida laboral desde la formalidad y ejercida a nivel profesional. Dos testimonios ejemplifican:

"Yo ya estoy pensionado, tengo problemas con mi pensión, pero yo relativamente me pensioné bien, yo trabajé y salí de una empresa, y yo era director de operaciones que es una posición muy alta (AM3, comunicación personal, 18 de junio de 2013)

"Yo fui maestra y estoy jubilada desde hace 10 años, gracias a Dios tengo mi pensión" (AM13, comunicación personal, 15 julio de 2013).

Se encontró que el factor común y determinante para que estos adultos mayores hayan tenido una vida laboral formal, fue el nivel educativo profesional alcanzado.

"Mi familia nunca fue rica pero tuve una mamá y un papá que les gustó mucho la educación de los hijos. Pues como todo trabajo que pasa un estudiante tuve que pasar, hay veces que uno no tiene pues la plata pa' la lonchera. O con un pasaje pero es como todo" (AM12, comunicación personal 9 de julio de 2013).

Por el contrario, en el caso de quienes carecen de pensión algunos nunca fueron a la escuela, otros alcanzaron como máximo grado de primaria segundo y cuarto, y los menos alcanzaron 
bachillerato, lo cual tuvo que ver con factores como falta de ingresos económicos, la mentalidad negativa de los padres acerca de la escuela, y el desinterés de los propios adultos en su infancia.

"Mi mamá era tan pobre y no me dio estudios. Yo aprendí a leer y a escribir por el periódico. Yo cogí el periódico, después de grande y pintaba las letras. Y las escribía y ya me decían qué decía ahí. Y ya aprendí a leer un poquito y a escribir, sin ortografía porque no sé ortografía" (AM4, comunicación personal, 21 de junio de 2013).

“Cómo te parece que estuve siete años de escuela y no pasé de primero!... Cuando terminé el año (mi mamá) iba y preguntaba a la maestra... Siete años y no pasé de primero..., lo poquito que sé me lo enseñó mi mamá. Yo era muy aficionado al juego, me pasaba con las bolsas llenas de cristal (canicas), de trompos y cosas. Yo escribo y leo pero mi mamá me enseñó" (AM7, comunicación personal, 26 de julio de 2013).

"No había sino hasta cuarto grado en esos pueblos de campo. Hasta eso estudié... en ese tiempo yo tenía 14 años. Mis padres no nos colaboraron. (Pero) mi padre decía 'ya usted sabe firmar, ya pa qué más, ¿pa qué va a estudiar?" (AM15, comunicación personal, 15 de julio de 2013).

Los adultos que trabajaron durante su vida en trabajos informales lo hicieron principalmente como vendedores o agricultores, casi siempre porque aprendieron de sus padres el oficio, por su estilo de vida y otros por decisión propia al no pensar en el futuro.

"Yo no trabajaba en la calle, yo trabajaba en una empresa pero la mamá de mi hija me embargó. Entonces me salí de la empresa y me quedé... muy muy mal porque yo debiera de haber... usted que sabe que un tiempo, como que no piensa en el día de mañana. Lo que uno piensa es en el de hoy, y yo no pensé en el de mañana y entonces la platica me la gasté... ya a la edad que yo tengo, no tengo empleo en nada, no tengo dinero. Por no querer hacer bien las cosas"... (AM 14, comunicación personal, 15 de julio de 2013)

Los especialistas señalan que si bien hoy se trabaja para que los futuros adultos mayores gocen de mejores condiciones, ya está aquí una generación de ancianos con múltiples condiciones desfavorables, entre ellas, la falta de una pensión.

"El adulto mayor de hoy (viene de) generaciones pasadas donde el nivel educativo era más bajo, el acceso a los servicios, la empleabilidad era más baja, los estados de salud más precarios, el control de las enfermedades... son generaciones pasadas que llegaron a este momento con menores tazas de pensiones, menores ingresos, más número de hijos, más dependencia económica"... (EI5, comunicación personal, 12 de julio de 2013).

En la actualidad existe un grado de seguridad económica producto del diseño de los sistemas de protección social de hace tres o cuatro décadas y así como de las características de los mercados de trabajo en aquel tiempo.3 Sin embargo, los especialistas reiteran que lo anterior produce que sean pocas las personas mayores que gozan de una jubilación laboral, lo cual hace que la vejez sea una etapa de inseguridad e incertidumbre, sin embargo, también consideran que se ha avanzado, pues el adulto mayor ya está en las agendas políticas y sociales, lo cual permitirá que las futuras generaciones de adultos mayores vivan en mejores condiciones que la actual.

"Si miramos las nuevas generaciones de personas mayores, que van a entrar, que pueden ser las personas que están en los 50 años y que están saliendo del mercado laboral, ellas sí van a tener una jubilación... van a tener unos ingresos para poder asegurar una vejez más tranquila" (EI5, comunicación personal, 12 de julio de 2013).

El apoyo familiar es uno de los mecanismos que generan seguridad económica para las personas mayores, y cumple con dos funciones básicas: asegurar la supervivencia, bienestar físico, alimento y vestido, así como proporcionar seguridad y los vínculos afectivos a sus miembros.2 En el adulto mayor, la familia se constituye en la red de apoyo social informal que le proporciona el mayor sustento físico y moral.16 En Colombia, la ayuda recibida de parte de miembros de la familia que viven dentro y fuera del país alcanza a apenas al 31\% de los adultos mayores que no trabajan.13 


\section{Transferencias por jubilaciones}

Otro tipo de transferencias son las pensiones por jubilación, que juntas en 2012 lograban la cobertura del 31\% de la población colombiana estimada en edad de jubilación, según el Ministerio del Trabajo.9,17 Según la Corte Consitucional de la República de Colombia, la pensión esun salario diferido del trabajador, fruto de su ahorro "En otras palabras, el pago de una pensión no es una dádiva súbita de la Nación, sino el simple reintegro que del ahorro constante durante largos años, es debido al trabajador".18 "El ingreso por pensión resulta ser la compensación económica al trabajo de los adultos mayores durante su historia laboral". 19

En Colombia, el sistema de pensiones cuenta con dos alternativas para que los trabajadores coticen o ahorren en búsqueda de lograr una pensión en su vejez: el régimen de prima media, administrado por el Estado y el régimen de ahorro individual solidario, administrado por fondos de pensión privados. Entre los dos están afiliados 17.2 de los 22 millones de trabajadores, sin embargo sólo el 7.7 millones ahorra activamente, revela el Ministerio de trabajo.17

En el caso concreto de la ciudad de Medellín, una encuesta realizada por la autoridad municipal estableció que el $85.9 \%$ de los trabajadores informales no realizan aportes para su pensión, lo cual aumenta su grado de vulnerabilidad "y deja prever que la carencia del derecho pensional, que hoy se muestra como una situación particular e individual, el Estado tendrá que asumir en forma colectiva en años futuros".20

\section{Transferencias por programas sociales}

Ninguno de los adultos mayores entrevistados goza de las transferencias del programa Colombia Mayor, porque no la necesitan, no califican o están en espera de una respuesta; los adultos que cuentan con ingresos fijos y suficientes para el costeo de sus gastos, manifestaron no necesitar dicho recurso. Por otro lado, los mayores que no cuentan con ingresos fijos y suficientes señalaron haber solicitado el beneficio, pero como respuesta no han obtenido la afirmación y mientras algunos se resignan, otros lo siguen intentando.
"Hace como tres años estuve haciendo vueltas para que me dieran ese auxilio, entregué papeles y como al año fui a preguntar y me dijeron que no los habían mandado a Bogotá. Y es que lo llevan a uno con mentiras, que no, que no los han mandado para Bogotá... esperé como tres meses, volví a preguntar y si me dijeron no, usted tiene un nivel muy alto, tiene que hacer otra encuesta para bajar puntos" (AM6, comunicación personal, 21 de junio de 2013).

Pese a lo difícil y costoso que les resulta el acudir constantemente a las instancias gubernamentales, los mayores buscan el beneficio pues dicho ingreso les sería muy útil, dadas las condiciones en que viven:

"Buscarla sí, yo metí los papeles del seguro, pero en primer lugar, dicen que por no ser cabeza de familia, no tengo derecho a nada. Es lo que me dicen a mí (AM14, comunicación personal, 15 de julio de 2013).

El gobierno colombiano ha optado por ser asistencialista $y$ ofrecer al adulto mayor programas que además de no generar soluciones de fondo a su entorno, se presentan como dádivas y no como el ejercicio de un derecho, señalan especialistas.

"Lo que está pidiendo el adulto mayor es que le den el derecho. Él no está diciendo en ningún momento que le den la limosna. ¿Por qué no le da el derecho, que lo tiene? Es necesario darle la oportunidad de que él gane dignamente... Porque es que él construyó una sociedad por espacio de 60 años y lo lógico es que disfrute de ese esfuerzo. Pero entonces lo vamos a premiar con 150 mil (pesos) cada dos meses. Es como decirle a ver si de pronto tenés derecho. No, es que el derecho ya se lo había ganado" (EI5, comunicación personal 12 de julio de 2013).

"Hay programas que facilitan subsidios económicos donde una persona recibe 75 dólares bimensuales, eso no es nada para lo que un ser humano necesita, digamos que hay respuestas paliativas" (EI3, comunicación personal, 21 de junio de 2013).

El programa Colombia Mayor, señalan, está lejos de ser la práctica de un derecho para el adulto 
mayor por su condición de viejo, pues sólo se dirige a personas de extrema pobreza. Además, no se trata de un beneficio constante y seguro.

"Pero quiénes tienen derecho a ello, aquellos que estén en la clasificación de beneficiarios de programas sociales Sisben 1 o 2, pero si a los dos meses que se vuelve a dar ese servicio esa persona está con mejores condiciones que otros que ingresan entonces es desplazado y ya no se lo garantizan, ellos no tienen garantizado el apoyo cada dos meses porque eso va a depender de que no lleguen otros en peores condiciones" (EI5, comunicación personal 12 de julio de 2013).

Añaden que es necesario que el gobierno, en conjunto con la sociedad y las instituciones continúen trabajando para que las futuras generaciones de adultos mayores tengan mejor condición de vida que la presente, lo cual será un beneficio para todos pues habrá ancianos autosuficientes que requerirán menor atención y recursos de parte de la sociedad y el estado.

"Es necesario empoderar mucho a los adultos mayores para lograr que lleguen con muchas mejores condiciones a la vejez y que no tengan que depender de nadie, si son autónomos, productivos, si tienen garantizadas ciertas condiciones de ingreso, seguridad económica, vivienda, mejores estados de salud van a exigir menos porque ellos se van a proveer de esa condición... tenemos que hacerlo con este grupo poblacional de adultos que en 20, 30 años van a ser adultos mayores para que cuando llegan allá no tengan que demandar" (EI5, comunicación personal, 12 de julio de 2013).

Señalan que pese a las carencias que tiene el sistema colombiano se cuenta con avances importantes y tangibles en la atención a los adultos mayores, como el establecimiento de comedores, apoyo económico a los CPSAM e incluso la creación del Cabildo Mayor, espacio desde donde los mayores participan y vigilan las decisiones que se tomen en torno a ellos.

También agregan que las instituciones educativas entregan a la sociedad gerontólogos y profesionales que además, de buscar la convivencia intergeneracional a fin de que el joven se familiarice con el adulto no como una persona incapaz, sino como otro miembro de la sociedad, agregan.

"Los recursos del estado siempre serán insuficientes, máxime si la población mayor va en aumento, pero hay servicios interesantes de larga estancia, servicios de salud, atención en salud domiciliaria, se han hecho avances... hay un aparataje muy interesante de lo que va a ser el procedimiento de envejecimiento y vejez" (EI4, comunicación personal, 26 junio de 2013)

"Yo pienso que tenemos en Medellín una población adulta mayor más empoderada. Pero como todo tiene un proceso... Yo pienso que el mayor en la ciudad de Medellín está siendo respetado, que el mayor en la ciudad de Medellín tiene otro posicionamiento, tiene otra conciencia, tiene otra visión, está reclamando sus derechos y lo está sabiendo hacer. Porque tenemos el Cabildo mayor con una buena organización, empoderados, empoderados, defendiendo, dejando claros planteamientos, desde el rescate de la dignidad del mayor" (EI3, comunicación personal, 14 de junio de 2013).

El apoyo económico mediante los programas gubernamentales es otra fuente importante de ingresos para los adultos mayores, sin embargo no garantizan su bienestar pues el déficit financiero institucional, la falta de cobertura total a la población envejecida y los bajos montos de las transferencias hacen que los adultos mayores busquen otras fuentes de ingreso.19

Mediante el programa Colombia Mayor, el gobierno nacional entrega una pensión bimensual a un millón de personas mayores de 65 años en situación de indigencia o pobreza extrema, lo que constituye el 20,1\% de los mayores de 60 años, y el 29,6\% de quienes tienen 65 y más, que es la edad solicitada para acceder a este programa. ${ }_{17} \mathrm{Se}$ trata de una pensión que consiste en una entrega de 150 mil pesos cada dos meses al beneficiario.21 Entre los requisitos están el pertenecer a los niveles 1 o 2 del Sistema de Identificación de Potenciales Beneficiarios de Programas Sociales (SISBEN) y no tener ingresos suficientes para subsistir, vivir solo y con un ingreso mensual que 
no supere medio salario mínimo legal vigente, vivir en la calle y de la caridad pública, vivir con la familia y que el ingreso familiar sea menor o igual al salario mínimo legal vigente, entre otros.22 $\mathrm{A}$ nivel Antioquia el apoyo alcanza a un total de 72,578 beneficiarios, y en Medellín a 47,500 • 23,24

El trabajo permite concluir que según la opinión de los entrevistados, los adultos mayores de Medellín no disfrutan de seguridad económica por razones diversas, entre ellas la carencia de ingresos, insuficiencia de los mismos, e incluso por la incertidumbre del porvenir. Los mayores medellinenses no tienen independencia económica, lo cual ha propiciado que algunos de ellos busquen un espacio en los Centros de Protección Social, ante la imposibilidad de sostenerse a sí mismos; de esta manera, son personas que si bien es cierto cuentan con los beneficios que estos lugares ofrecen, también manifiestan sentirse limitados para el ejercicio de sus actividades -debido a horarios y reglamentosy preocupados por no contar con un ingreso que les permita adquirir artículos o alimentos que se les antojan, por lo que no se puede hablar de una independencia ni de autosuficiencia.

Los adultos mayores de Medellín, Colombia, viven en escenarios distintos, sin embargo tienen en común la necesidad de contar con un ingreso económico para la cobertura de necesidades básicas, las cuales varían según cada individuo. La mayor parte de los adultos carece de ingresos fijos, lo cual les limita en sus decisiones, y mientras algunos se resignan, otros luchan por mantenerse incluso desde la informalidad, donde la situación es de vivir al día, conscientes de que cada vez se envejecerá más y a la par aumentará la dificultad para seguir laborando. Por otro lado, prácticamente no existen espacios donde se oferten empleos formales para los mayores, como tampoco los mecanismos para lograr que este grupo ejerza su derecho al trabajo.

Las transferencias familiares como fuente de ingreso principal para los adultos mayores son un medio menos común que el trabajo; quienes dependen totalmente de ellas son personas que no laboran, pero tampoco cuentan con otro tipo de ingreso. No se puede hablar de una seguridad económica a partir de las transferencias familiares ya que éstas son irregulares e insuficientes. Por otro lado, hay casos en que los mayores reciben ingresos por parte de miembros de su círculo familiar, pero como complemento a otras entradas.

A pesar de que la ley en Colombia expresa que la familia tiene obligación conjunta con el estado y la sociedad de apoyar al adulto mayor vulnerable, las familias no están siendo responsables en cumplir con la parte que les corresponde, al igual que el resto de los involucrados en la citada ley.

La pensión por jubilación es un derecho que no todos los adultos mayores pueden ejercer debido a las condiciones en que laboraron durante su vida. Quienes gozan de este beneficio pueden descansar en él y logran una vida tranquila; lo contrario sucede con aquellos adultos mayores que no están jubilados, pues tienen que continuar trabajando o están a expensas de lo que la sociedad, las familias y el gobierno les aporte.

Hoy existen opciones de jubilación futura para los trabajadores que se desempeñan desde la informalidad, ya que pueden hacer aportaciones voluntarias para acceder en el futuro a una pensión. Sin embargo, el porcentaje de informales que hace uso de este beneficio es apenas poco más del $10 \%$, lo que pudiera reflejar la falta de concientización sobre el tema.

Las transferencias económicas provenientes del gobierno resultan insuficientes en cifra y alcance para los adultos de Medellín. Los lineamientos del programa Colombia Mayor establecen que el adulto debe estar en condiciones extremas de pobreza para recibir un apoyo de 150 mil pesos cada dos meses. El tener como requisitos para la obtención del subsidio un ingreso familiar no mayor a la mitad salario mínimo, evidencia las condiciones que viven muchos adultos mayores de esta localidad, lo cual expone la incapacidad del Estado por cumplir sus propias leyes, que le señalan como uno de los responsables de generar los mecanismos para que los adultos mayores tengan una vida digna. A pesar de lo anterior, se percibe en el gobierno y la sociedad de Medellín una intención por mejorar las condiciones de vida 
de los adultos mayores, la existencia de leyes a favor de él, los recursos económicos para la ejecución de programas, el voluntariado, la apertura de espacios como el Cabildo Mayor, y generación de profesionistas para atender a este grupo social desde las instituciones educativas hablan de que se está en camino hacia una mejor realidad para este sector.

Conflicto de intereses. Ninguno declarado por los autores.

\section{Referencias}

1. UNFPA, CELADE. Política Nacional en favor de las personas mayores en Panamá. [Internet]. 2004 [citado 5 de junio de 2014]. Disponible en: http://www.eclac.org/celade/noticias/paginas/3/196 53/PoliticaNacionalPanama.pdf

2. CEPAL. Seguridad económica y pobreza en la vejez: tensiones, expresiones y desafíos. Notas de Población No. 83 [Internet]. 2006 [citado 13 de julio de 2013]. Disponible en: http://www.eclac.cl/publicaciones/xml/9/30029/lcg 2340-P4.pdf

3. Ramos M, Vera D, Cárdenas M, Fondo de Población de las Naciones Unidas, Las personas adultas mayores y su contribución a la lucha contra la pobreza. [Internet]. 2009 [citado 15 de julio de 2013]. Disponible en: http://www.unfpa.org.pe/publicaciones/publicacione speru/MIMDES-Personas-Adultas-Mayores.pdf

4. Mena J. Seguridad económica, desarrollo humano y pobreza. Documento preliminar. (Centro de Estudios Sociales y de Opinión Pública) [Internet]. 2010 [citado 6 de junio de 2013]. Disponible en: http://www3.diputados.gob.mx/camara/content/do wnload/248452/720686/file/Seguridad_economica_ desarrollo_pobreza_docto101.pdf

5. Alles M. Autoempleo: una salida frente al desempleo. Buenos Aires: Ediciones Granica; 2005.

6. Páez T. Capacidad emprendedora e informalidad en el campo de la economía. Investigación y Postgrado. 2008.

7. Yanes R. Una ciudad para todas las edades». En Conapo, El envejecimiento demográfico en México: retos y perspectivas. México. 1999: 395-407.

8. Departamento Administrativo de Planeación. Encuesta Calidad de Vida 2010. Medellín. 2011.

9. Fondo de Población de las Naciones Unidas, UNFPA. Las personas adultas mayores y su contribución a la lucha contra la pobreza. 2009.

10. Consejo Nacional de Población. Envejecimiento demográfico en México: retos y perspectivas. Por una sociedad para todas las edades.

11. Dulcey-Ruiz E, Arrubla D. Serie de estudios a profundidad ENDS 1990-2010. Envejecimiento y vejez en Colombia 2010.

12. Asociación Probienestar de la Familia Colombiana Profamilia. Encuesta Nacional de Demografía y Salud
ENDS 2010 [Internet]. [citado 5 de junio de 2014]. Disponible en: http://dhsprogram.com/pubs/pdf/FR246/FR246.pdf

13. República de Colombia. Ministerio de Protección Social. Política Nacional de Envejecimiento y Vejez 2007-2019 [Internet]. 2007 [citado 17 de julio de 2013]. Disponible en: http://www.funlibre.org/documentos/diplorecyam/ PoliticaNalDeEnvejecimientoyVejez.pdf

14. Giraldo-Ocampo C, Cardona-Arango D. Ser viejo en Colombia tiene su costo laboral. Investigaciones Andinas. 2010; 12(21).

15. Banco de la República. Informalidad laboral en las áreas urbanas de Colombia [Internet]. [citado 5 de junio de 2014]. Disponible en: http://www.banrep.gov.co/sites/default/files/publica ciones/archivos/DTSER-164.pdf

16. Cardona D, Estrada A, Agudelo H. Envejecer nos «toca» a todos [Internet]. [citado 5 de junio de 2014]. Disponible en:

http://cienciagora.com.co/imgs2012/imagenes/Envej ecer_nos_toca_a_todos_Medellin_2003.pdf

17. República de Colombia. Ministerio del Trabajo. Nuevo modelo de protección para la vejez [Internet]. [citado 5 de junio de 2014]. Disponible en: http://webcache.googleusercontent.com/search?q=cac he:a5blvT-

xoE0J:www.mintrabajo.gov.co/component/docman/do c_download/1256-abece-nuevo-modelo-de-proteccionpara-la-

vejez.html+\&cd=1\&hl=es\&ct=clnk\&gl=co\&client=firefo $\mathrm{x}-\mathrm{a}$

18. República de Colombia. Corte Constitucional. Consulta de la Norma: SENTENCIA C-546 DE 1992 REF: Procesos Nos. D-023 y D-041 [Internet]. [citado 5 de junio de 2014]. Disponible en: http://www.alcaldiabogota.gov.co/sisjur/normas/Nor ma1.jsp?i=9317\#3.3.1.-14

19. Millán-León B. Factores asociados a la participación laboral de los adultos mayores mexiquenses. Papeles Poblac. 2010;16(64):93-121

20. Personería de Medellín. Informe sobre la situación de los derechos humanos en la ciudad de Medellín 2012 [Internet]. [citado 5 de junio de 2014]. Disponible en: http://www.personeriamedellin.gov.co/documentos/I NFORME_D1.pdf

21. República de Colombia. Ministerio del Trabajo. Cobertura total de «Colombia Mayor» para San Andrés [Internet]. [citado 5 de junio de 2014]. Disponible en: http://www.mintrabajo.gov.co/febrero-2013/1514cobertura-total-de-qcolombia-mayorq-para-sanandres.html

22. Consorcio Colombia Mayor. Programa Colombia Mayor [Internet]. [citado 5 de junio de 2014]. Disponible en: http://colombiamayor.co/programa_colombia_mayor. html

23. Ministerio de Trabajo. Presidente y MinTrabajo entregan 14.578 nuevos subsidios a adultos mayores en Antioquia. Bogotá: MinTrabajo.

24. Gil L. En 2013, la Alcaldía de Medellín aumentó la cobertura y los recursos para los Adultos Mayores. 